\title{
QUANTIFICATION OF DEPTH OF ANESTHESIA BY MEANS OF ADAPTIVE CALCULATION OF CORRELATION DIMENSION PARAMETERS
}

\author{
BEHZAD AHMADI*,†, ${ }^{*}$, BAHAREH ZAGHARI ${ }^{*, \dagger}$, \\ RASSOUL AMIRFATTAHI ${ }^{*},{ }^{\dagger}$ and MOJTABA MANSOURI ${ }^{\dagger}$ \\ *Digital Signal Processing Research Lab \\ Department of Electrical and Computer Engineering \\ Isfahan University of Technology \\ Isfahan, 84156-83111, Iran \\ ${ }^{\dagger}$ Medical Image and Signal Processing Research Center \\ Isfahan University of Medical Sciences, Isfahan, Iran \\ ¥behzadahmadi@ec.iut.ac.ir \\ ‡behzad.ahmadi.m@gmail.com
}

Received March 17, 2009

Accepted April 27, 2009

\begin{abstract}
This paper proposes an approach for quantifying Depth of Anesthesia (DOA) based on correlation dimension (D2) of electroencephalogram (EEG). The single-channel EEG data was captured in both ICU and operating room while different anesthetic drugs, including propofol and isoflurane, were used. Correlation dimension was computed using various optimized parameters in order to achieve the maximum sensitivity to anesthetic drug effects and to enable real time computation. For better analysis, application of adaptive segmentation on EEG signal for estimating DOA was evaluated and compared to fixed segmentation, too. Prediction probability (PK) was used as a measure of correlation between the predictors and BIS index to evaluate the proposed methods. Appropriate correlation between DOA and correlation dimension is achieved while choosing (D2) parameters adaptively in comparison to fixed parameters due to the nonstationary nature of EEG signal.
\end{abstract}

Keywords: Adaptive Segmentation; Bispectral Index; Depth of Anesthesia; Correlation Dimension. 


\section{INTRODUCTION}

In the operating room, the anesthesiologist is essential in providing optimal working conditions to surgeons, and in ensuring patient safety and comfort. However, patient awareness during surgery with the rate of $1: 1000^{1}$ and overdosing with anesthetic agents are major clinical concerns of anesthesia. Therefore, the necessity to assess and monitor the depth of anesthesia (DOA) is obvious. In conventional methods, DOA is measured based on the monitoring of several physiological signals such as respiration pattern, blood pressure, body temperature, tearing, sweating and heart rate, ${ }^{2}$ even though these signals are affected indirectly by anesthetic agents. On the other hand, these agents have significant effects on the electroencephalogram (EEG) waveform. Hence, it is advocated that the EEG can provide a reliable basis for deriving a surrogate measurement of anesthesia.

In recent years, the anesthesia community has witnessed the development of a number of EEGbased algorithms of consciousness. Several of the above methods are available that have recently been reviewed by Freye et $a l .{ }^{3}$ and Jameson et al. ${ }^{4}$

The earliest methods were based on the FFT analysis of EEG signals. These approaches tend to find parameters that describe spectrum characteristics. Peak power frequency (PPF), median power frequency (MPF), and spectral edge frequency (SEF) have been the first descriptors in this field. Another parameter extracted from spectrum was the ratio of power in two empirically derived frequency bands. ${ }^{5}$ In a work presented by Traast et $a .^{6}$ the power of EEG in different frequency bands was determined and the results indicate pronounced changes in EEG during emergence from propofol/sufentanil total intravenous anesthesia.

Ferenets et al. ${ }^{7}$ analyzed the performance of several new measures based on the regularity and complexity of the EEG signal. These measures consist of spectral entropy (SpEn), approximate entropy (ApEn), Higuchi fractal dimension (HFD) and Lempel-Ziv complexity (LZC). Their results show superior ability of the mentioned measures to predict DOA. Due to the arguments presented in their paper it is not feasible to point out "the best" EEG measure for the assessment of the depth of sedation Their results indicate that the measures sensitive to both the power spectrum as well as the amplitude distribution, i.e., the ApEn, LZC and HFD, perform slightly better than the other two tested measures. In the case of their tested measures, they recommend window length of $20 \mathrm{~s}$.

Application of neural networks (NN) in estimating DOA is reviewed by Robert et al. ${ }^{8}$ They examined a large number of EEG derived parameters as NN inputs including spectral, entropy, complexity, bicoherence, wavelet transformation derived, autoregressive modeling and hemodynamic parameters as well as a great NN topology such as MLP and Self-Organizing networks. Finally, they recommended a two hidden layers MLP model or an ART model in which their weights are updated after training phase continuously. Moreover the use of qualitative parameters, besides quantitative ones, as network inputs is recommended. In a recent work by Lalitha et al. ${ }^{9}$ non-linear chaotic features and neural network classifiers are used to detect anesthetic depth levels. Chaotic features consist of correlation dimension, Lyapunov exponent (LE) and Hurst exponent (HE) are used as features and two neural network models, i.e., multi-layer perceptron network (feed forward model) and Elman network (feedback model) are used for classification. Their experimental results show that the Lyapunov exponent feature with Elman network yields an overall accuracy of $99 \%$ in detecting the anesthetic depth levels.

According to various mentioned methods, different EEG monitors have been developed. The Narcotrend $^{\mathrm{TM}}$ monitor (Monitor Technik, Bad Bramsted, Germany) that is based on pattern recognition of the raw EEG and classifies the EEG into different stages, introduces a dimensionless Narcotrend ${ }^{\mathrm{TM}}$ index from 100 (awake) to 0 (electrical silence). The algorithm uses parameters such as amplitude measures, autoregressive modeling, fast Fourier transform (FFT) and spectral parameters. ${ }^{10}$ The SEDLine ${ }^{\mathrm{TM}}$ EEG monitor, capable of calculating of $\mathrm{PSI}^{\mathrm{TM}}$ index, uses the shift in power between the frontal and occipital areas. The mathematical analysis includes EEG power, frequency and coherence between bilateral brain regions. ${ }^{11}$ Datex-Ohmeda ${ }^{\mathrm{TM}} \mathrm{s} / 5$ entropy Module uses entropy of EEG waves to predict $\mathrm{DOA}^{4}$ and finally BIS ${ }^{\mathrm{TM}}$ (Aspect Medical Systems, Newton, $\mathrm{MA}$ ), that is the first monitor in the marketplace and has become the benchmark comparator for all other monitors, introduces the BIS ${ }^{\mathrm{TM}}$ index (that is a unit-less number between 100 and 0) as a DOA indicator based on combination of spectral, bispectral and temporal analysis. ${ }^{5}$ Approximately 
450 peer-reviewed publications between 1990 and 2006 have been examined the effectiveness, accuracy and usefulness, both clinical and economical, of the BIS $^{\mathrm{TM}}$ monitor. ${ }^{4}$

The interpretation of the EEG is complex since it is a reflection of the brain state, the effects of the surgical procedure and the influence of anesthetic drugs. Anesthetic agents have different effects on the EEG and do not represent a straightforward solution concerning the monitoring of depth of anesthesia. Thus, EEG processing using linear methods such as power of frequency bands, spectral edge frequency, etc. did not solve the problem. Nevertheless, the ideas that the EEG informs the anesthesiologist directly about depth of anesthesia and the inadequacy of linear measures to assess this depth under different circumstances were the main motivation to investigate the application of non-linear mathematics to the EEG. Consequently, nonlinear dynamical analysis has emerged a novel method for the study of complex systems during the past few decades. Lyapunov exponent, Hurst exponent, fractal dimension, information dimension, box dimension, and correlation dimension are some of the methods by which the complexity of a system or a signal could be quantified. Correlation dimension is by the most popular due to its computational efficiency compared to other fractal dimensions such as the information dimension, capacity dimension, and pulse dimension. ${ }^{12}$

A significant step forward was made by Grassberger and Procaccia ${ }^{13}$ who showed how the so-called correlation dimension could be used to estimate and bind the fractal dimension of the strange attractor associated with the nonlinear time signal at hand. The method was immediately applied to many different types of time series such as financial time series, ${ }^{14}$ physiological recordings. ${ }^{12,15-17}$ Specifically, in Ref. 18, the application of correlation dimension to determine anesthetic drug effect was described. Watt et al. demonstrated the changes of phase space trajectories and dimensionality as a result of changed depth of anesthesia. ${ }^{19}$ Later, Widman et al. investigated a modified version of $D 2$, the nonlinear correlation index $D^{*}$, as a measure of depth of sevoflurane anesthesia. ${ }^{20}$ Lee et al. found $D 2$ to serve as a better index for the depth of halothane anesthesia in the rat compared to beta-power and median power frequency. ${ }^{21}$ All of the previous studies revealed the potency of $D 2$ or derived measures to measure the level of consciousness and to support the presumption that shifting from full consciousness to unconsciousness results in more and more left out autonomic processes. Due to the ability of correlation dimension in expressing this complexity in a single number, it looks very attractive for the purpose of an indicator of depth of anesthesia.

The aim of this paper is to quantify the dynamic of the system, which is affected by anesthetic drugs. Thus, correlation dimension of EEG signal is calculated using optimized adaptive parameters. Our aim is to extract the required parameters out of EEG signal by means of adaptive algorithms and methods. It is further assumed that when DOA is changing gradually, the EEG signal is composed of consecutive stationary segments which could be detected by adaptive segmentation methods. Consequently, application of adaptive segmentation in computing correlation dimension is investigated, too. Specifically, we focus on finding better estimators of our selected sub-parameters. Electroencephalograms were collected from patients undergoing surgery. Correlation dimension using different methods and parameters was extracted through different fixed and adaptive windowing methods. Afterwards, correlation between these parameters and BIS index was evaluated via proper statistical analysis. The improved correlation proposes the utilization of the adaptive methods in BIS algorithm.

The organization of the rest of the paper is as follows: In Sec. 2, methods and materials are described. The results are presented in Sec. 3, and Sec. 4 includes the final conclusion and discussion.

\section{METHODOLOGY}

In this section, the experiment, the data acquisition, and the data analysis are described.

\subsection{Patients}

Following the approval of the ethical committee of the medical school, eight coronary artery bypass graft surgery candidates were selected (six males, two females, of average age 56.2 years and the average weight of $68.3 \mathrm{~kg}$ ) and written informed consents were obtained from all selected subjects. Inclusion criteria were absence of neurological disorders such as cerebrovascular accidents and convulsions. Pre-operative neurological complications (such as cerebral emboli and convulsion) caused exclusion from the study. The anesthesiologist performed pre-operative evaluation on the 
day before surgery. For anxiolysis, the patients were pre-medicated by intramuscular morphine $0.1 \mathrm{mg} / \mathrm{kg}$ and promethazine $0.5 \mathrm{mg} / \mathrm{kg}, 30$ minutes before being transfered to operating room. After arrival in the operating room, electrocardiogram, pulse oxymetry, depth of anesthesia, and invasive blood pressure monitoring were established. The BIS-QUATTRO sensorTM (Aspect Medical Systems, Newton, MA) was applied to the forehead of the patients before induction of anesthesia. After pre-oxygenation with O2, eight patients were anesthetized in the same manner by intravenous thiopental sodium $(5 \mathrm{mg} / \mathrm{kg})$, pancuronium bromide $(0.1 \mathrm{mg} / \mathrm{kg})$, fentanyl $(5 \mu \mathrm{g} / \mathrm{kg})$, and lidocaine $(1.5 \mathrm{mg} / \mathrm{kg})$. After the induction of anesthesia and until cardiopulmonary bypass beginning, anesthesia was continued by administration of isoflurane (1 MAC), morphine $(0.2 \mathrm{mg} / \mathrm{kg})$ and $\mathrm{O} 2(100 \%)$. During coronary artery bypass grafting under $\mathrm{CPB}$, patients were anesthetized by propofol (50$150 \mu \mathrm{g} / \mathrm{kg} / \mathrm{min})$ under BIS control (40-60) and O2 $(80 \%)$. For organ protection during CPB, patients were undergone mild hypothermia $\left(31-33^{\circ} \mathrm{C}\right)$. After coronary artery bypass grafting, patients rewarming, and obtaining standard CPB separation criteria, the patients were gradually weaned from $\mathrm{CPB}$. After separation from CPB, anesthesia was continued by isoflurane (1 MAC) and O2 (100\%) administration to the end of surgery. After surgery, patients were transported to ICU under portable monitoring And manual ventilation. In the ICU, mechanical ventilation with $60 \%$ fractioned inspired oxygen and standard homodynamic monitoring were continued. In ICU and until complete recovery, the sedative regimen was intravenous morphine $(2 \mathrm{mg})$ if needed. In this study, the raw EEG data and relative BIS index were collected during the whole period of operation from operating room arrival to complete recovery in the intensive care unit.

\subsection{Data Acquisition}

The EEG signal was collected by using a BISQUATTRO Sensor TM that was composed of selfadhering flexible bands holding four electrodes, applied to the forehead with a frontal-temporal montage.

The used EEG lead was Fpz-At1, and the reference lead was placed at FP1. The sensor was connected to a BIS-X-P Monitor and all binary data packets containing raw EEG data wave signals and BIS index which is converted to binary format using an A/D converter operating with $128 \mathrm{~Hz}$ sampling frequency were recorded via an RS232 interface on a laptop using a Bi-spectrum analyzer developed with C++ Builder by Satoshi Hagihira. ${ }^{22}$ The algorithms that are presented in this study were tested on these raw EEG signals.

The sensor was attached to the patient's forehead at the beginning of anesthesia process and the data was collected continuously until patients' awakeness at ICU. Therefore, in this study a large amount of EEG data with their BIS index was collected for each patient. Some other events such as changes of anesthesia regimen, intubations, applying CPB and transferring to ICU were recorded. Because of short acting time of thiopental sodium (approximately $15-20 \mathrm{~s}$ ), this part of EEG data was not analyzed.

\subsection{Phase Space}

Let $x(i)$ for $i=1,2,3, \ldots, N$ denotes an observable process generated by an unknown or unobservable system. Following Brock, ${ }^{23} x(i)$ is said to have deterministic explanation if:

$$
\begin{gathered}
F: M \rightarrow M \\
s(t+1)=F(s(t)),
\end{gathered}
$$

where $F$ is an unknown smoth map, and $m$ is a $d$-dimensional manifold and:

$$
\begin{gathered}
h: M \rightarrow \Re \\
x(t)=h(s(t)),
\end{gathered}
$$

where $h$ is an unknown smoth map. Then, according to Takens theorem ${ }^{24}$ and Packard et al. ${ }^{25}$ for an adequate choice of parameters $E_{m}$ and $\tau$, there exists a function $G$ such as:

$$
\begin{gathered}
G: \Re^{E_{m}} \rightarrow \Re^{E_{m}} \\
X(t+\tau)=G(X(t)),
\end{gathered}
$$

with

$$
X(i)=\left(x(i), x(i+\tau), \ldots, x\left(i+\left(E_{m}-1\right) \tau\right)\right)
$$

and $E_{m}$ being the embedding dimension and $\tau$ the time delay which is usually fixed to one. The above vector is suggested by Takens ${ }^{24}$ for the extraction of information in the dynamics of chaos by reconstructing the phase space (state space) from time series of signal variable.

Thus, if $x(i)$ is a deterministic time series, then for any pair of points $(y(i), y(j))$ and for an adequate choice of $E_{m}$, there exists arbitrary small $a$ 
and $\delta>0$ so that:

$$
\begin{aligned}
& \text { if }\|y(i)-y(j)\|<a \\
& \text { then }\|G(y(i))-G(y(j))\| \ll \delta \text {, }
\end{aligned}
$$

where $\|a-b\|$ being the distances between the vectors $a$ and $b$. This property means that the images of close points are closed in the phase space.

This construction produces a trajectory in $E_{m}$ dimensional space. We slide a window of length $E_{m}$ through the time series and stack the $E_{m}$ dimensional vector column-wise into matrix $Y$ :

$Y=\left[\begin{array}{ccc}x(k) & \cdots & x\left(N-\left(E_{m}-1\right) \tau\right) \\ x(k+\tau) & \cdots & x\left(N-\left(E_{m}-2\right) \tau\right) \\ \vdots & & \vdots \\ x\left(k+\left(E_{m}-1\right) \tau\right) & \cdots & x\left(N-\left(E_{m}-E_{m}\right) \tau\right)\end{array}\right]$.

Each row of the above matrix is now a point in $E_{m}$-dimensional reconstructed phase space. This "phase space continuity" is the characteristic of deterministic processes; it can be used to distinguish random number from chaotic dynamics and to make short term prediction of chaotic time series. ${ }^{26}$ Figure 1 displays two and three-dimensional mapping of reconstructed phase space based on $Y$.

\subsection{Correlation Dimension Estimation}

The Grassberger and Proccacia algorithm using Theiler $\operatorname{method}^{27,28}$ considers spatial correlation

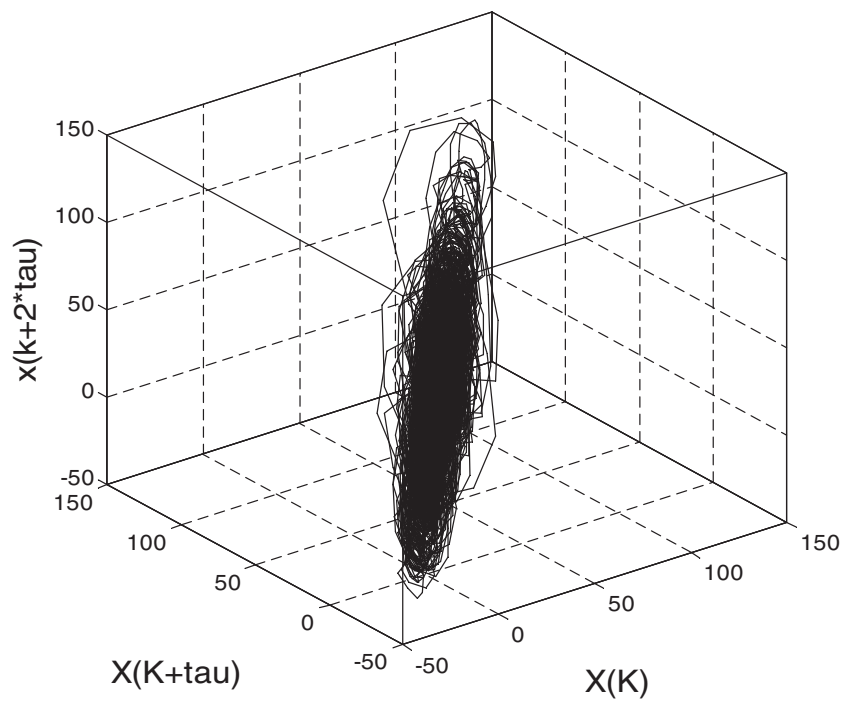

Fig. 1 A time lag embedding: One of the data sets is used in the calculation together with the time lag embedding in two and three dimensions. (The time lag is $\tau=1$.) between pairs of points on a reconstructed attractor. For an $E_{m}$-dimensional phase space, the modified correlation integral $C(r)$ is defined as ${ }^{13}$

$$
\begin{aligned}
C(r)= & \frac{2}{(m+1)(m)} \\
& \times \sum_{i=1}^{m} \sum_{j=i}^{m-i} \Theta(r-\|X(i)-X(j)\|),
\end{aligned}
$$

where $m=N-\left(E_{m}-1\right) \tau$ is the number of embedded points in an $E_{m}$-dimensional space, $N$ is the length of data series, $r$ is the radius of sphere centered on $x(i)$ or its box size, and $\Theta(x)$ is the heaviside step function as follows

$$
\Theta(x)= \begin{cases}1 & x \geq 0 \\ 0 & x<0\end{cases}
$$

$r$ is changed to obtain better correlation dimension. If $C(r)$ scales like

$$
C(r)=r^{v},
$$

then, $v$ is called the correlation dimension of the time series $x$ or equivalently the slop of the log-log plot of $C(r)$ versus $r$.

Other questions are the proper choice of $\tau$ (time delay), $E_{m}$ (embedding dimension), the range of $r$ value in which the slope can be regarded as a good estimator of fractal dimension. ${ }^{29}$ Furthermore, the optimum way of extracting the above values from the numerical values of $C(r)$ will be pointed. In Sec. 2.5, $r$ is determined using different approaches and the corresponding D2 values would be computed.

\subsection{Determination of $r$}

In the literature, the parameter $r$ has been treated to be fixed in a population or at least for a subject. ${ }^{30}$ Therefore, a raw idea is to apply fixed $r s$ and calculate the correlation dimension. In this part of study, $r$ is changed manually between 1 and 20 and D2 is calculated. The corresponding D2 value is named "fixed $r$ ".

The idea of the Theiler's algorithm ${ }^{27}$ is to apportion the data points into an $E_{m}$-dimensional "boxes" of size $r^{*}$. Thus, only distances between points that are either in the same box or neighboring boxes are computed. This algorithm (which uses $\left.r^{*}\right)$ is referred to as Standard Box Assisted Correlation Algorithm (SBAC). ${ }^{31}$ 


\subsubsection{Standard box assisted correlation algorithm}

The box size $r^{*}$ is the only parameter over which the user has full control in the box assisted correlation algorithm. $r^{*}$ could be found by using the definition of correlation integral $C(r)$ according to the following equation

$$
m=\frac{1}{2} m^{2} \cdot C\left(r^{*}\right),
$$

where $m=N-\left(E_{m}-1\right) \tau$. By using the approximation $C\left(r^{*}\right) \approx r^{* v}$, one gets:

$$
r_{T}=\left(\frac{2}{m}\right)^{\frac{1}{v}}
$$

where $r_{T}$ denotes the box size suggested in Theiler's paper. ${ }^{27}$ In this study, correlation dimension is estimated using both $r_{T}$ and $r^{*}$. The estimated D2 values are named " $r_{T}$ " and " $r$ ", respectively.

\subsubsection{Mean distance between neighbors}

In the correlation dimension theory, $r$ is known to be the radial distance around each reference point $X(i)$. Another idea is to put $r$ as the mean distance between each $X(i)$ and its neighbor $X^{N N}(i)$ as follows

$$
r_{\text {mean }}=\operatorname{mean}\left\|X(i)-X^{N N}(i)\right\| .
$$

In this study, correlation dimension is estimated using $r_{\text {mean }}$. We nominate the D2 values computed by means of this algorithm the "mean distance".

\subsubsection{Fraction of standard deviation of the signal}

Another idea is to put $r$ as the fraction of the standard deviation of the EEG signal for which the correlation dimension is calculated.

$$
r=k \sigma,
$$

where $k$ is ranging from 0.5 to 0.7 and $\sigma$ is the standard deviation of the EEG signal in the specified window. D2 values extracted out of this algorithm are named " $k=0.5 \mathrm{std}$ " and " $k=0.7 \mathrm{std",}$ respectively.

\subsection{Determination of Delay Time}

The delay time $\tau$ is commonly determined by using the autocorrelation function (ACF) method (via finding the place where ACF first attains zeros or below a small value, e.g. 0.2 or 0.1 ), or by using mutual Information (MI) method (via finding the place where MI first attains a minimum) ${ }^{32}$ In this study, $\tau$ is changed manually from one to ten.

The result shows that smaller $\tau s$ would yield in better correlation between D2 and BIS index. This confirms the results obtained from mutual information algorithm (see Ref. 33). The results obtained in Ref. 33 have yielded in smaller $\tau s$, too (approximately one).

\subsection{Embedding Dimension Estimation}

In order to find the optimal embedding dimension $\left(E_{m}\right)$, the False Nearest Neighbor method (FNN) is considered. ${ }^{34} \mathrm{FNN}$ method is based on the Euclidean distance between apparent neighbors in the phase space. It examines the fraction of nearest neighbors as a function of the embedding dimension to determine the necessary global dimension to unfold an attractor. Thus, the minimum embedding dimension is found when most of the nearest neighbors do not move apart significantly in the next higher dimensional embedding. Each vector $X(i)$ has the nearest neighbor in dimension $E_{m}$ which is named $X^{N N}(i)$. Let $R_{E m}(i)$ be the distance between vectors $x(i)$ and its neighbors:

$$
\begin{aligned}
R_{E m}(i) & =\left\|X(i)-X^{N N}(i)\right\| \\
& =\sqrt{\sum_{j=0}^{E_{m}}\left(x(i+j \tau)-x^{N N}(i+j \tau)\right)^{2}} .
\end{aligned}
$$

A threshold $R_{T}$ is required to decide when neighbors are false. The neighbors that fulfill:

$$
R_{T}<\frac{\left|x\left(i+E_{m} \tau\right)-x^{N N}\left(i+E_{m} \tau\right)\right|}{R_{E_{m}}(i)}=R
$$

at time point $i$ are considered false. By applying Equation (15) for $E_{m}=2,3,4, \ldots$ until the fraction of points for which $R>R_{T}$ is negligible, the optimal embedding dimension could be clarified. In this study, $E_{m}$ is selected by computing the percentage of false nearest neighbors. Applying the above algorithm, the embedding dimension could be observed from Fig. 2. According to Fig. 2, the embedding 


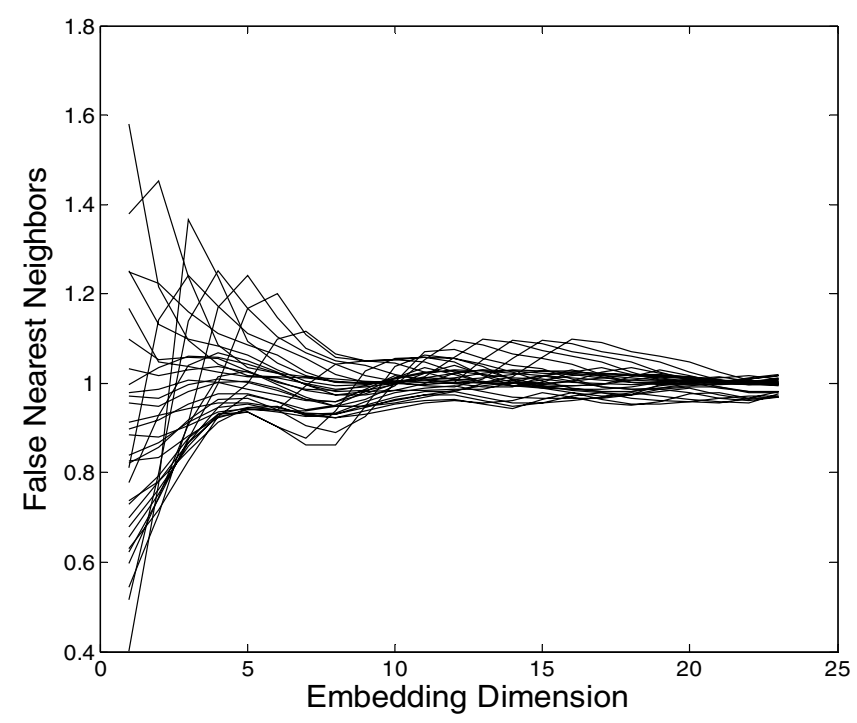

Fig. 2 The fraction of $x(i)$ as a function of $E_{m}$ for the embedding of the bivariate $x(i)$ and $x^{N N}(i)$ time series.

dimension value is set to 10 for calculating correlation dimension.

\subsection{Adaptive Segmentation of EEG Data}

In the previous sections and with regards to other works in this area, ${ }^{7-9}$ fixed window length $(L=$ $20 \mathrm{~s}$ ) were applied to EEG signals.

It would be desirable to adapt the analysis window to changes in the given signal, allowing the window to be as long as possible while the signal remains stationary, and to start a new window at the exact instant when the signal changes its characteristics. ${ }^{35}$ In order to perform the described approach, two adaptive windowing methods have been used: adaptive variance detection and ACF (Auto-Correlation Function) distance methods. In both approaches, a reference window is extracted at the beginning of each scan, and the given EEG signal is observed through a moving window. In each of the above algorithms, the performance of the reference window length $L=1$ second has been evaluated.

In adaptive variance detection method, a segment boundary is drawn when the variance of the moving window becomes $k$ times greater or larger than the variance of the reference window. By considering the changes of EEG variance, $k=7$ and $k=10$ are tested and finally $k=10$ was considered better for applying the above procedure. (The D2 value obtained via using this method of windowing is named "variance".)
On the other hand, for implementing ACF distance method, let $\phi_{R}(k)$ be the ACF of the reference window at the beginning of a new segmentation step, where $k$ is the lag or delay. Let $\phi_{T}(n, k)$ be the ACF of the test and sliding window positioned at time instant $n$. A normalized power distance $d_{p}(n)$ between ACFs is computed as:

$$
d_{p}(n)=\frac{\left|\sqrt{\phi_{T}(n, 0)}-\sqrt{\phi_{R}(0)}\right|}{\min \left\{\sqrt{\phi_{T}(n, 0)}, \sqrt{\phi_{R}(0)}\right\}} .
$$

The condition where $d_{p}(n)$ becomes larger than a specific threshold, $T h_{P}$, is considered to represent a significant change in $\mathrm{ACF}$, and is used to mark a segment boundary. ${ }^{35}$ Again, due to the variation of $d_{p}(n)$ values, three different $T h_{P} s$ (25000, 30000 , and 35000), are tested and $T h_{P}=30000$ is used to evaluate the described approach. (The D2 value obtained by using this method of windowing is named "ACF").

\subsection{Statistical Analysis}

The correlation between BIS index and the extracted sub-parameters was investigated with the model-independent Prediction Probability $(\mathrm{Pk}) .{ }^{36}$ As a nonparametric measure, the $\mathrm{Pk}$ is independent of scale units and does not require knowledge of underlying distributions or efforts to linearize or otherwise transform scales. A Pk value of 1 means that the predicting variables ("mean distance", "fixed $r ", " k=0.5 \mathrm{std}$ ", " $k=0.7 \mathrm{std} ", " r_{T} ", " r *$, "variance", and "ACF") always predict the value of the predicted variable (e.g., BIS index) correctly. $\mathrm{Pk}$ value of 0.5 means that predictors predict no better than only by chance. The $\mathrm{Pk}$ values were calculated on a spreadsheet using the Excel 2003 software program and the PKMACRO written by Warren Smith. ${ }^{36}$ In the case of inverse proportionality between indicator and indicated parameters, the actual measured $\mathrm{Pk}$ value is $1-\mathrm{Pk}$. Another statistical analysis used in this study was ordinal logistic regression. This regression examines the relationship between one or more predictors and an ordinal response. The index that determines the efficiency of this regression model is called "Concordant", which shows the percentage of values predicted successfully with the model.

Our results are presented in three different groups; during propofol and isoflurane anesthesia in operating room and in ICU under sedative regimens. By fixing $\tau$ to one and calculating $E_{m}$ via 
FNN method, D2 is computed using eight different parameters and the correlation between our extracted parameters ("mean distance", "fixed $r$ ", " $k=0.5 \operatorname{std} ", " k=0.7 \mathrm{std} ", " r_{T} "$, " $r *$, "variance", and "ACF") and BIS index was calculated via proper statistical analysis. In order to apply logistic regression analysis, the total BIS index range was divided into six non-overlapping groups: 0-25, 25-40, 40-50, 50-60, 60-80, and 80-100.Then, the best model was determined to identify how good a predictor could predict the BIS level.

\section{RESULTS AND CONCLUSION}

The upcoming figures are the scattering plots of the BIS index (measured from the patients) versus correlation dimension.

According to Figs. 3-5, D2 (correlation dimension) shows certain tendency to decrease with increasing depth of anesthesia using various measures and algorithms described in this study. The figures are depicted for ICU, isoflurane and propofol groups. Of course, the linearity of different parameters will be analyzed further.

The above behavior is acceptable due to the fact that correlation dimension is known as a measure of complexity and EEG is become less complex while patients are anesthetized. More coherencies between BIS index and D2 value could be observed from Figs. 3 and 4 in comparison to Fig. 5. This is due to the different capabilities of the proposed parameters in estimating BIS index.

The correlation between the extracted parameters and BIS index (Bispectral index) is measured

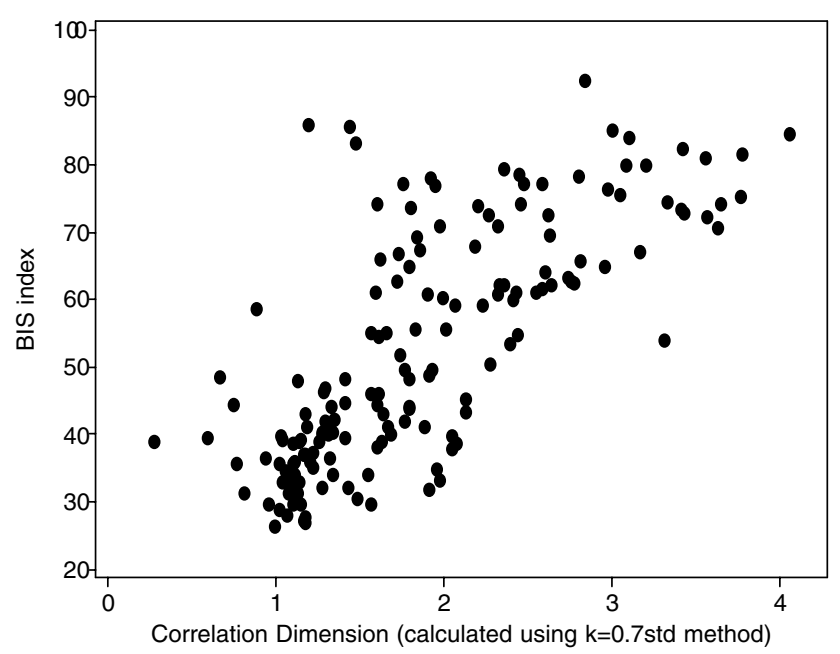

Fig. 3 BIS index versus correlation dimension using " $k=$ 0.7 std" method.

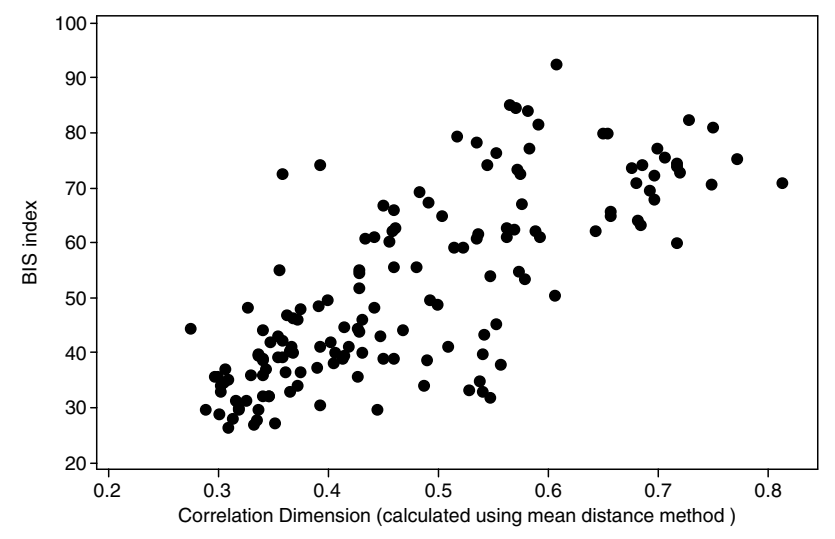

Fig. 4 BIS index versus correlation dimension using mean distance method.

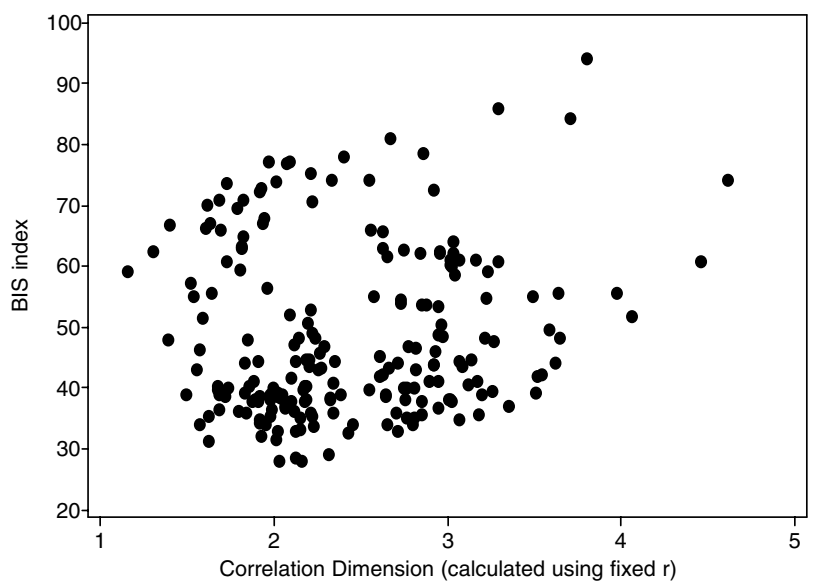

Fig. 5 BIS index versus correlation dimension using fixed $r$ method.

by means of the statistical methods described in the previous section and the results are presented in Figs. 6-9.

Figure 6, compares the efficiency of different parameters in predicting BIS index. In this figure, prediction probability is used as a statistical measure. The results show that the calculating correlation dimension using the proposed parameters (i.e. " $k=0.7 \mathrm{std} ", " k=0.5 \mathrm{std}$ ", and " $r_{T}$ ") would yield in a better estimator of BIS index in the ICU group. Other parameters are approximately the same in predicting DOA. Application of adaptive segmentation was not so successful, although it is superior to fixed segmentation in some aspects.

With comprehension of prediction probability values for different methods in calculation of correlation dimension in isoflurane group (Fig. 7), it would be claimed that standard deviation method is better than the others. Like ICU, adaptive segmentation is not capable of improving the assessment 


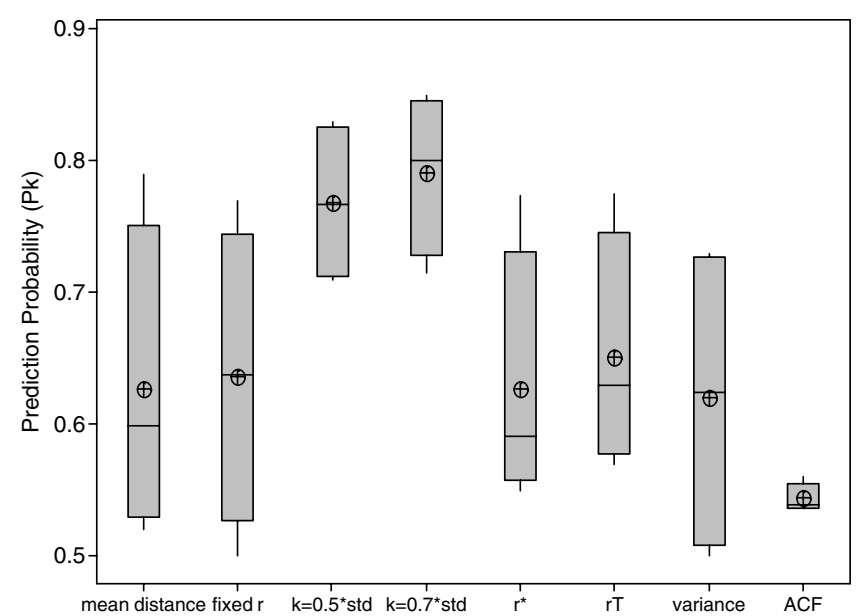

Fig. 6 Correlation between the extracted parameters and BIS index measured in ICU group.

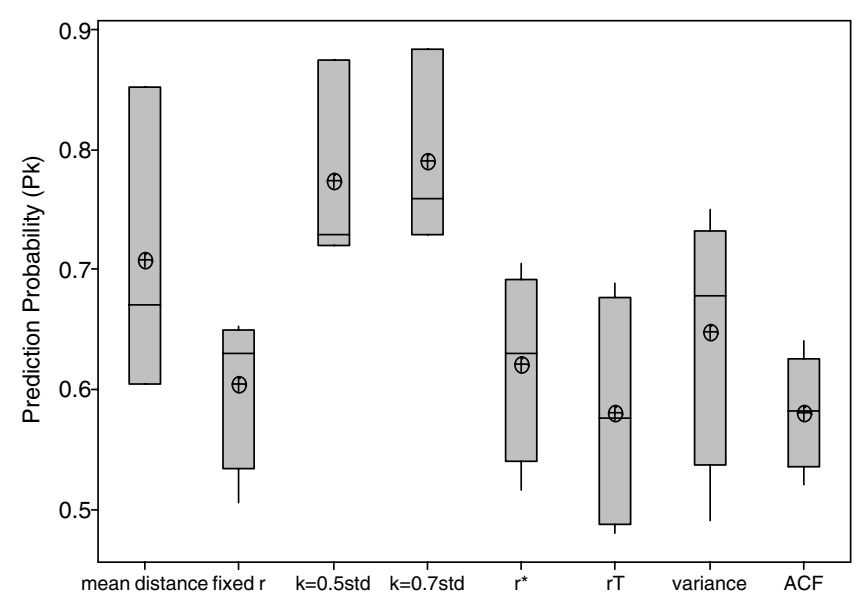

Fig. 7 Correlation between the extracted parameters and BIS index measured in isoflurane group.

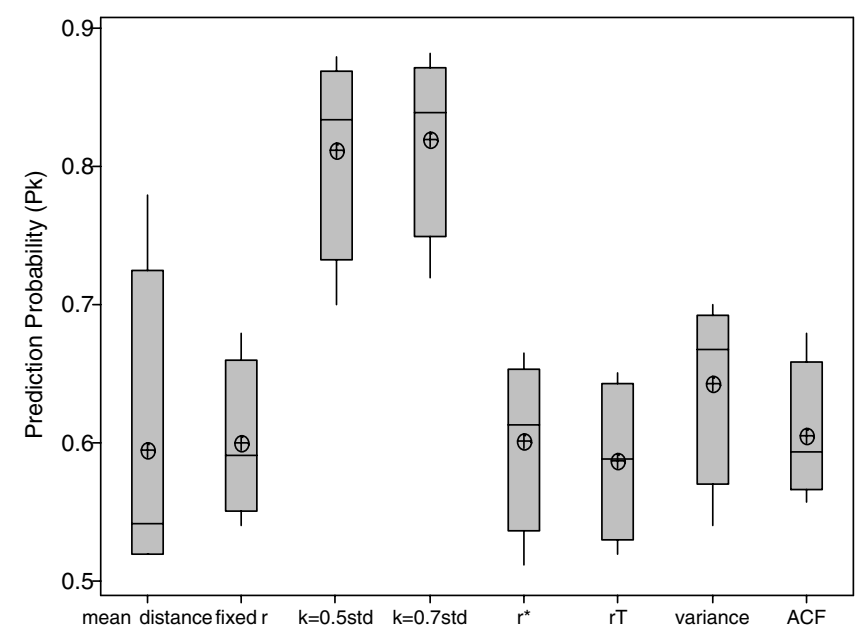

Fig. 8 Correlation between the extracted parameters and BIS index measured in propofol group.

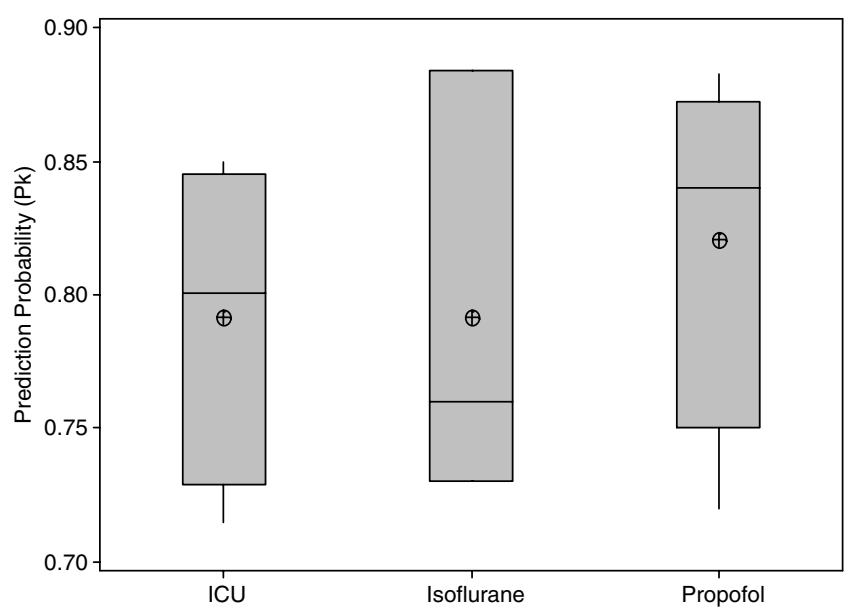

Fig. 9 Comparing the performance of D2 in predicting BIS index using " $k=0.7 \mathrm{std}$ " method in different groups.

of DOA based on calculation of D2. In isoflurane group, the "mean distance" parameter is more capable of estimating BIS index than " $r_{T}$ " and " $r$ " parameters. The "fixed $r$ " parameter has the least correlation with BIS index.

The $\mathrm{Pk}$ values obtained in propofol group (Fig. 8) reveals that computing D2 based on " $k=0.7 \mathrm{std}$ " and " $k=0.5 \mathrm{std"}$ " parameters would result in better DOA estimators. The only difference between the result in ICU and propofol is the sensible superiority of adaptive segmentation methods (such as variance) in comparison to other parameters (except " $k=0.7$ std" and " $k=0.5$ std") in propofol.

Finally, in order to compare the results in different groups, " $k=0.7 \mathrm{std}$ " is chosen in each of the groups and the results are shown in a single plot (Fig. 9).

According to Fig. 9, our findings shows that the results are slightly better in propfol group in comparison to other groups while using " $k=0.7 \mathrm{std}$ " based on calculation of D2.

\section{DISCUSSION}

Based on adaptive calculation of correlation dimension parameters, a new approach was presented to estimate BIS index. In this study, raw EEG signals with relative BIS index of eight patients undergoing coronary artery bypass graft surgery have been acquired. Then, different algorithms (using fixed and adaptive segmentation methods) were applied to the recorded EEG signals. In order to investigate the capability of the extracted parameters in 
predicting BIS index, appropriate statistical analyses were used in three different groups.

The first and foremost outcome was that D2 values were directly proportional to BIS index, which confirms the results mentioned in similar studies. ${ }^{20,21}$ Different parameters which are effective in computing correlation dimension were investigated. First of all, the embedding dimension was extracted out of the EEG signals. Then, the main focus was put on calculating the parameter " $r$ " adaptively based on EEG signal. The results show the high correlation between D2 and BIS index while computing " $r$ " via the proposed standard deviation method. The results of applying adaptive segmentation were better than "fixed $r$ ". This was also true while using SBAC algorithm for calculating " $r$ ", but the latter was not as powerful as the standard deviation method. Our other proposed method ("mean distance") reveals high correlation with BIS in some of the groups (especially propofol). Finally, D2 performs more satisfactory in propofol group for estimating BIS index. In conclusion, the modified D2 parameter shows high correlation with BIS index due to its ability in quantifying EEG signal complexity. In conclusion, the findings show the potential for applications of concepts derived from the theory of nonlinear dynamics, even if little can be assumed about the process under investigation.

Furthermore, in order to improve the accuracy of the depth of anesthesia estimation, comparison against sedation scales (such as OAA/S) and drug levels is needed. The reason is that BIS is not equal to depth of anesthesia but needs to be validated for DOA assessment itself.

The work reported is preliminary. Although the results are significant, a wider patient population is necessary for better evaluation. Therefore, obtained claims and outcomes should be confirmed by working on a larger group of patients and drugs and through various surgeries. To sum up, the approaches proposed in this study based on the application of adaptive algorithms, including ACF and variance methods for segmentation, SBAC, mean distance, and standard deviation methods for determination of sphere radius $(r)$, and FNN method for determination of embedding dimension, improve the performance of correlation dimension in estimating BIS index. According to various studies, the above parameters are the main parameters used in calculating correlation dimension. Thus, the proposed segmentation methods may enhance the capability of correlation dimension in estimating depth of anesthesia.

\section{REFERENCES}

1. J. G. Jones, Perception and memory during general anesthesia, Br. J. Anaesth. 73 (1994) 31-37.

2. R. D. Miller, Miller's Anesthesia, 6th edn. (Elsevier Churchill Livingstone, 2005) pp. 1227-1264.

3. E. Freye and J. V. Levy, Cerebral monitoring in the operating room and the intensive care unit: an introductory for the clinician and a guide for the novice wanting to open a window to the brain. Part I: the electroencephalogram, J. Clin. Monit. Comput. 19 (2005) 1-76.

4. L. C. Jameson and T. B. Sloan, Using EEG to monitor anesthesia drug effects during surgery, J. Clin. Monit. Comput. 20 (2006) 445-472.

5. I. J. Rampil, A primer for EEG signal processing in anesthesia, Anesthesiology 89 (1998) 980-1002.

6. H. S. Traast and C. J. Kalkman, Electroencephalographic characteristics of emergence from propofol/sufentanil total intravenous anesthesia, Anesth. Analg. 81 (1995) 366-371.

7. R. Ferenets, T. Lipping, A. Anier, V. Jäntti, S. Melto and S. Hovilehto, Comparison of entropy and complexity measures for the assessment of depth of sedation, IEEE Trans. Biomed. Eng. 53(6) (2006) 1067-1077.

8. C. Robert, P. Karasinski, C. D. Arreto and J. F. Gaudy, Monitoring anesthesia using neural networks: a survey, J. Clin. Monit. Comput. 17 (2002) 259-267.

9. V. Lalitha and C. Eswaran, Automated detection of anesthetic depth levels using chaotic features with artificial neural networks, J. Med. Syst. 31 (2007) $445-452$.

10. R. Bender, B. Schultz and U. Grouven, Classification of EEG signals into general stages of anesthesia in real time using autoregressive models, in Conf. Proc. 16th Ann. Conf. Ges. Klass. (University of Dortmund, 1992) pp. 443-452.

11. D. R. Drover, H. J Lemmens, E. T. Pierce, G. Plourde, G. Loyd, E. Ornstein, L. S. Prichep, R. J. Chabot and L. Gugino, Patient State Index: titration of delivery and recovery from propofol, alfentanil, and nitrous oxide anesthesia, Anesthesiology 97 (2002) 82-89.

12. B. J. West, Fractal Physiology and Chaos in Medicine, Studies of Nonlinear Phenomena in Life Sciences, Vol. 1 (World Scientific, Singapore, 1990).

13. P. Grassberger and I. Procaccia, Characterization of strange attractors, Phys. Rev. Lett. 50 (1983) 346349 . 
14. D. Hsieh, Chaos and nonlinear dynamics: applications to financial markets, J. Finance 46 (1991) 1839-1877.

15. J. Ulbikas and A. Cenys, Nonlinear dynamics methods in EEG investigations, in Advances in Synergetics, Vol. 1 (Belarusian State University Press, Minsk, 1994) pp. 110-120.

16. I. M. Irurzun, P. Bergero, M. C. Cordero, M. M. Defeo, J. L. Vicente and E. E. Mola, Non-linear properties of R-R distributions as a measure of heart rate variability, Chaos Solitons Fractals 16 (2003) 699-708.

17. P. Pascolo, F. Barazza and R. Carniel, Considerations on the application of the chaos paradigm to describe the postural sway, Chaos Solitons Fractals 27 (2006) 1339-1346.

18. G. Mayer-Kress, S. P. Layne, S. H. Koslow, A. J. Mandell and M. F. Shlesinger, Perspectives in Biomedical Dynamics and Theoretical Medicine (Annals of the New York Academy of Sciences, New York, USA, 1987) pp. 62-87.

19. R. C. Watt and S. R. Hameroff, Phase space electroencephalography (EEG): a new mode of intraoperative EEG analysis, J. Clin. Monit. Comput. 5 (1988) 3-13.

20. G. Widman, T. Schreiber, B. Rehberg, A. Hoerof and C. E. Elger, Quantification of depth of anesthesia by nonlinear time series analysis of brain electrical activity, Phys. Rev. E 62 (2000) 4898-4903.

21. M. G. Lee, E. J. Park, J. M. Choi and M. H. Yoon, Electroencephalographic correlation dimension changes with depth of halothane, Kor. J. Physiol. Pharmacol. 3 (1999) 491-499.

22. S. Hagihira, M. Takashina, T. Mori, T. Mashimo and I. Yoshiya, Practical issues in bispectral analysis of electroencephalographic signals, Anesth. Analg. 93 (2001) 966-970.

23. W. A. Brock, Distinguishing random and determining the minimum embedding dimension of scalar time series, Physica D 110 (1986) 43-50.

24. F. Takens, Detecting Strange Attractors in Fluid Turbulence, Springer Lecture Notes in Mathematics, Vol. 898 (Springer, Berlin, 1981) pp. 366-381.
25. N. H. Packard, J. P. Crutchfield, J. D. Farmer and R. S. Shaw, Geometry from a time series, Phys. Rev. Lett. 45 (1980) 712-716.

26. J. D. Farmer and J. J Sidorowich, Predicting chaotic time series, Phys. Rev. Lett. 59 (1981) 845-848.

27. J. Theiler, Efficient algorithm for estimating the correlation dimension from a set of discrete point, Phys. Rev. A 36(9) (1987) 4456-4462.

28. J. Theiler, Spurious dimension from correlation algorithms applied to limited time series data, Phys. Rev. A 34(3) (1986) 2427-2432.

29. J. Theiler, Statistical error in a chord estimator of correlation dimension: the rule of five, Int. J. Bifurcat. Chaos 3 (1993) 765-771.

30. M. Koskinen, T. Seppanen, S. Tong, S. Mustola and N. V. Thakor, Monotonicity of approximate entropy during transition from awareness to unresponsiveness due to propofol anesthetic induction, IEEE Trans. Biomed. Eng. 53(4) (2006) 669-675.

31. A. Bueno Orovio and V. M. Pérez García, Enhanced box and prism assisted algorithms for computing the correlation dimension, Chaos Solitons Fractals 34 (2007) 509-518.

32. A. M. Fraser and H. L. Swinney, Independent coordinate for strange attractors from mutual information, Phys. Rev. A 33 (1986) 1134-1140.

33. W. W. Nga, U. S. Panu and W. C. Lennox, Chaosbased analytical techniques for daily extreme hydrological observations, J. Hydrol. 342(1-2) (2007) $17-41$.

34. M. B. Kennel, R. Brown and H. D. I. Abarbanel, Determining embedding dimension for phase space reconstruction using a geometrical construction, Phys. Rev. A 45 (1992) 3403-3411.

35. R. M. Rangayyan, Biomedical Signal Analysis: A Case-Study Approach (IEEE Press, New Jersey, 2001) pp. 405-416

36. W. D. Smith, R. C. Dutton and N. T. Smith, Measuring the performance of anesthetic depth indicators, Anesthesiology 84(1) (1996) 38-51. 